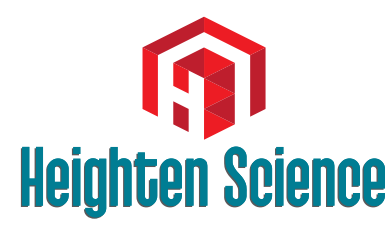

P U B L I C A T I O N S Corporation

\title{
Oral Candida colonization in HIV- infected patients: Species and antifungal susceptibility in Tripoli/Libya
}

\author{
Mohamed $\mathrm{H}^{1}$, Krema Z1 , Mokthar E¹, Ellabib $\mathrm{M}^{1 *}$, El Magrahi $\mathrm{H}^{2}$ \\ and Eshwika $\mathrm{A}^{3}$ \\ 'Department of Medical Microbiology and Immunology, Faculty of Medicine, Tripoli University, \\ Tripoli, Libya \\ ${ }^{2}$ Department of Medical Laboratory Science, Faculty of Medical Technology, Tripoli, Libya \\ ${ }^{3}$ Department of Microbiology, Faculty of Pharmacy, Zawia University, Libya
}

\begin{abstract}
*Address for Correspondence: Ellabib M, Department of Medical Microbiology and Immunology, Faculty of Medicine, Tripoli University, P.O. Box 13497, Tripoli, Libya, Tel: 0021-8925 857619; Email: ellabib@mail.com

Submitted: 18 July 2018

Approved: 02 August 2018

Published: 03 August 2018

Copyright: @ 2018 Mohamed H, et al. This is an open access article distributed under the Creative Commons Attribution License, which permits unrestricted use, distribution, and reproduction in any medium, provided the original work is properly cited.
\end{abstract}

Keywords: Candida; HIV; Azole resistance

Check for updates

\section{Abstract}

Introduction: Candidiasis is more frequent in human immunodeficiency virus (HIV)-infected patients and knowledge about the distribution and antifungal susceptibility of oral Candida species is important for effective management of candidiasis.

Material and Methods: An oral rinses sample collected from hundred HIV-infected patients with and without clinical evidence of oral candidiasis in this study at the Infectious Department/Tripoli Medical Center, Libya. Species identified by standard phenotypic and conventional methods and in vitro susceptibility testing of the yeast isolates to antifungals were performed using the Disc diffusion method protocol as recommended by the Clinical Laboratory Scientific Institute.

Results: Oral Candida colonization is detected in all patients with and without clinical syndromes, and Candida albicans were accounted for (74\%), C. dubliniensis (11\%) and C. glabrata (6\%). A high proportion of Candida species ( $42 \%$ ) showed decreased susceptibility to fluconazole. Among C., albicans more than $30 \%$ of isolate were resistant to most new azole antifungal including fluconazole, itraconazole, posoconazole and voriconazole.

Conclusions: A significant number of oral Candida species particular Candida albicans exhibiting decreased susceptibility to fluconazole were isolated from colonized HIV-infected individual, given the high incidence and severity of fungal infections in HIV-infected individuals. The results of this study reinforce the importance of antifungal susceptibility testing, which contributes to the therapeutic strategies and highlights the need for continuous surveillance of Candida colonization in this population.

\section{Introduction}

Oral candidiasis or oral thrush is the most common opportunistic yeast infection caused Candida species of the mucous membranes of the mouth [1,2]. C. albicans is the most common species of yeast isolated from patients with oral candidiasis [3]. The incidence of opportunistic infections due to Candida albicans and other Candida species has been increasing [4]. Oral Candidiasis is the most common HIV related oral lesion and most patients were infected with a strain originally present as a commensal of the oral cavity [5]. The low absolute CD4+T-lymphocyte count were traditionally been cited as the greatest risk factor for the development of oropharyngeal candidiasis and current guidelines suggest increased risk once CD4+T lymphocyte counts fall below 200 cells/ $\mu \mathrm{l}[6,7]$. The first step in the development of a candida infection is colonization of the mucocutaneous surfaces, in this respect HIV infection is not only associated with increased colonization rates but also with the development of overt disease during the course of HIV infection [6]. The rate of Candida infection is related to the $\mathrm{CD} 4$ counts of the patient, which in turn depends on the use of Anti-retroviral 
treatment [7]. HIV-positive patients carry more and a greater variety of yeasts than HIV-negative subjects. The prolonged management of oral candidiasis in HIV patients might cause the development of drug resistance candidiasis [8]. Although the introduction of antiretroviral therapy (ART) has had a major, impact on the infectious complications of AIDS [9], Candidiasis still remains a common opportunistic infection in HIV-infected patients [6]. This study aimed mainly to determine the prevalence of oral candida colonization and infection in HIV positive patients and their susceptibility profile to a wide range of common usably antifungal drugs.

\section{Materials and Methods}

\section{Study design}

This study has conducted between the mycology laboratory at the department of medical microbiology and infectious diseases department, Tripoli Medical Center, Libya from May 2017 to May 2018. The ethical research committee gave ethical approval for the study. HIV seropositive patients with or without clinical symptoms for oral candidiasis attending infectious disease department for routine follow up check have been enrolled in this study. Demographic data have taken for each patient including age, sex, clinical signs, and symptoms suggestive of oral candidiasis examined to ascertain the presence of candida infection. CD4 count and patient on ART therapy or not taking ART or taking antifungal drugs for the last three months also have been included.

\section{Study population}

Fifty-nine male and forty-one HIV positive patients were included that attend ART clinic of Infectious Diseases department for routine follow-up. The patients included in the study were from different location, $58(58 \%)$ with average 50 years old followed by 30 years average age group 33(33\%) and more than 60 years old $9(9 \%)$.

\section{Sample collection culture and identifications}

One hundred HIV patient with and without symptomatic oral candidiasis disease were enrolled in this study. A patient's information sheet including information such as age, gender, oral condition, CD4 count, antifungal and antiretroviral therapy (ART) were filled for each patient. Patient who were on ART were taking nucleoside reverse transcriptase inhibitors including efavirenz 600mg one capsule/at night and Combivir cap (lamivudine+zidovudine /12hr). Samples obtained by an oral concentrated rinse in which the mouth washed for 30 second with $10 \mathrm{ml}$ of sterile water. The mouthwash liquid then deposited in conical tubes and stored in an insulated container until microbiological processing. The sample suspension was washed three times in sterilized $0.1 \mathrm{M}$ phosphate-buffered saline $\mathrm{pH} 7.4$ (PBS) by centrifugation at $3500 \mathrm{rpm}$, the pellet then re suspended in $1 \mathrm{~mL}$ of PBS, and $100 \mu \mathrm{l}$ aliquots were inoculated by a spiral plating system onto the surface of Sabouraud's dextrose agar with antibiotic. Plates was incubated at $30^{\circ} \mathrm{C}$ for $24-72 \mathrm{~h}$ growth assessed by enumeration of colonies and expressed as candida colony forming units per $\mathrm{mL}$ (cfu/mL-1) of rinse [10]. Yeasts species identified by germ-tube production, micromorphology, and chlamydospores production on corn meal agar plus $1 \%$ Tween 80 . All isolates show germ tube test and chlamydospores test positive on corn meal agar and Tween 80 was identified as $C$. albicans or $C$. dubliniensis. Sunflower seed agar and xylose hypertonic media [11,12] was used to differentiate $C$. albicans from $C$. dubliniensis. Candida non-albicans species were identified by API 20C AUX (bioMérieux's).

\section{Antifungal susceptibility testing}

Five distinct colonies of approximately $1 \mathrm{~mm}$ from each 24-hour old culture grew on Sabouraud dextrose agar incubated at $35 \pm 2^{\circ} \mathrm{C}$ suspended in $5 \mathrm{ml}$ of sterile $0.85 \%$ saline. The resulting suspension was adjusted to the turbidity to yield $1 \times 10^{6}-5 \times 10^{6}$ cells/ml (i.e., 0.5 McFarland standard). A sterile cotton swab moistened with the 
inoculum suspension was used to inoculate a $90 \mathrm{~mm}$ diameter plate containing Mueller Hinton agar supplemented with $2 \%$ glucose and $0.5 \mu \mathrm{g} / \mathrm{ml}$ methylene blue (GM-MH agar medium) $[13,14]$. The plates allowed drying for 5-15 min before antifungalembedded disks placed in the center of the agar. The following antifungal disks were purchased from Liofilchem, Italy: amphotericin B ( $20 \mu \mathrm{g})$, caspofungin (5 $\mu \mathrm{g})$, clotrimazole $(50 \mu \mathrm{g})$, econazole $(10 \mu \mathrm{g})$, fluconazole $(25 \mu \mathrm{g}$ and $100 \mu \mathrm{g})$, fluocytosine $(1 \mu \mathrm{g})$, itraconazole ( $50 \mu \mathrm{g})$, ketoconazole $(10 \mu \mathrm{g})$, miconazole $(10 \mu \mathrm{g})$, nystatin $(10$ $100 \mathrm{IU})$, posoconazole $(5 \mu \mathrm{g})$, and voriconazole $(1 \mu \mathrm{g})$. The plates then incubated for 18 - 24 hours at $37 \pm 2^{\circ} \mathrm{C}$ and the slowly growing isolates read after 48 hours incubation. Inhibition zone size interpreted according to CLSI document criteria for susceptible and resistant [14]. The following strains were used as quality control are C. albicans ATCC 90028 and C. parapsilosis ATCC 22019 strains for fluconazole and voriconazole disk diffusion testing, according to CLSI guidelines [15].

\section{Results}

Out of the 100 patients examined for oral colonization of candidiasis, 100 patients $(100 \%)$ yielded Candida species. Males accounted for the higher prevalence 59 (59\%) compared to $41(41 \%)$ females. The age group 40-60 years accounted for the highest prevalence 58 (58\%) followed by 20-40 age group 33(33\%) and more than 60 years 9 (9\%). The result show that patient taking ART and their CD4+count above 200 are lese colonized by high density with candida species $(<100 \mathrm{CFU} / \mathrm{ml})$ than patient not taking ART and lower CD4+count, Patient data including CD4+T lymphocyte count and oral colonization density shown in Table 1.

All patients found colonized with different candida species and detectable growths were produce by all isolates after incubation for 24-48 hours. Calbicans had the highest frequency among all the species with a total number of 74 (74\%). The non-albicans species and their frequencies are Cdubliniensis 11 (11\%), C. glabrata 6 (6\%), Ctropicalis, C parapsilosis 3 each (3\%), C krusei 2 (2\%), and C. catenulata 1 (1\%). Candida albicans were most isolated species from both patients on ART and not on ART therapy. The antifungal susceptibility test as determined by the disk diffusion method for isolates presented in Table 2. All Candida isolates tested were susceptible to amphotericin B and nystatin, caspofungin. C. albicans were shown to be highly resistant to fluconazole, itraconazole, posoconazole and voriconazole while, $C$. dubliniensis, C. glabrate and other Candida species isolates were more susceptible. All isolates including C. albicans were more susceptible to clotrimazole, econazole, miconazole and ketoconazole. All isolates except $C$. glabrata, $C$. parapsilosis and $C$. catenulata were determined as more resistant to fluocytosine. C. albicans strains isolated from patient on ART therapy and high CD+4 T-lymphocytes cell count were less resistant to fluconazole, itraconazole, posoconazole, voriconazole and other antifungal than strains isolated from patient not taking ART as shown in Table 3.

Table 1: Demographic data of HIV-seropositive patients with Candida colonization and its correlation with ART and CD4+count.

\begin{tabular}{|c|c|c|c|c|c|}
\hline Demographic Data & & & Oral-Candidal colonization & & \\
\hline 3 & CD4 count & NP & $10-90 \mathrm{CFU} / \mathrm{ml}$ & $>10^{5} \mathrm{CFU} / \mathrm{ml}$ & Total \\
\hline Patients on $\operatorname{ARE}(n=52)$ & $<200$ & 1 & 1 & 0 & 1 \\
\hline & $200-500$ & 17 & 6 & 11 & 17 \\
\hline 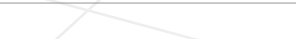 & $>500$ & 34 & 26 & 8 & 34 \\
\hline Total number of patients & & 52 & 33 & 19 & 52 \\
\hline 0 & & & $\lambda$ & & \\
\hline Patient not on ART $(n=48)$ & $<200$ & 25 & 6 & 19 & 25 \\
\hline 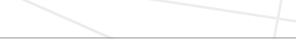 & $200-500$ & 18 & 8 & 10 & 18 \\
\hline & $>500$ & 5 & 2 & 3 & 5 \\
\hline Total number of patients & 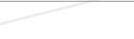 & 48 & 16 & 32 & 48 \\
\hline \multicolumn{6}{|c|}{ NP (number of patients); ART (antiretroviral therapy). } \\
\hline
\end{tabular}




\begin{tabular}{|c|c|c|c|c|c|c|c|c|c|c|c|c|c|}
\hline \multicolumn{14}{|c|}{$\%$ of isolates susceptible to antifungal drug } \\
\hline Candida species (n) & $\begin{array}{c}\text { AMB } \\
20\end{array}$ & FLU25 & FLI100 & POS5 & NY10 & CL050 & ITC50 & AFY1 & v01 & CAS5 & KCA10 & MCL10 & ECN10 \\
\hline \multicolumn{14}{|l|}{$\begin{array}{l}\text { Candida albicans } \\
\qquad(\mathrm{n}=74)\end{array}$} \\
\hline $\mathrm{s}$ & 100 & 41.9 & 44.6 & 50 & 91.9 & 100 & 54.1 & 44.6 & 43.2 & 96 & 43.2 & 54.1 & 31.1 \\
\hline 1 & 0 & 1.4 & 1.4 & 0 & 8.1 & 0 & 0 & 25.7 & $1 . .4$ & 2.7 & 14.9 & 33.8 & 36.5 \\
\hline $\mathrm{R}$ & 0 & 56.7 & 54.1 & 44.6 & 0 & 0 & 46 & 29.7 & 55.4 & 1.4 & 41.9 & 12.2 & 32.4 \\
\hline \multicolumn{14}{|l|}{$\begin{array}{l}\text { Candida dubliniensis } \\
\qquad(\mathrm{n}=11)\end{array}$} \\
\hline $\mathrm{s}$ & 100 & 90.9 & 90.9 & 100 & 100 & 100 & 90.9 & 54.6 & 90.9 & 81.8 & 90.9 & 100 & 90.9 \\
\hline 1 & 0 & 0 & 0 & 0 & 0 & 0 & 0 & 18.2 & 0 & 9.1 & 0 & 0 & 9.1 \\
\hline $\mathrm{R}$ & 0 & 9.1 & 9.1 & 0 & 0 & 0 & 9.1 & 27.3 & 9.1 & 9.1 & 9.1 & 0 & 0 \\
\hline \multicolumn{14}{|l|}{ Candida glabrata (n-6) } \\
\hline $\mathrm{s}$ & 100 & 100 & 100 & 66.7 & 83.3 & 100 & 100 & 83.3 & 100 & 100 & 100 & 100 & 100 \\
\hline 1 & 0 & 0 & 0 & 33.3 & 16.7 & 0 & 0 & 16.7 & 0 & 0 & 0 & 0 & 0 \\
\hline $\mathrm{R}$ & 0 & 0 & 0 & 0 & 0 & 0 & 0 & 0 & 0 & 0 & 0 & 0 & 0 \\
\hline \multicolumn{14}{|l|}{ C. tropicalis $(n=3)$} \\
\hline $\mathrm{s}$ & 100 & 100 & 100 & 100 & 100 & 100 & 100 & 33.3 & 100 & 66.7 & 100 & 100 & 33.3 \\
\hline 1 & 0 & 0 & 0 & 0 & 0 & 0 & 0 & 33.3 & 0 & 33.3 & 0 & 0 & 33.3 \\
\hline $\mathrm{R}$ & 0 & 0 & 0 & 0 & 0 & 0 & 0 & 33.3 & 0 & 0 & 0 & 0 & 33.3 \\
\hline \multicolumn{14}{|l|}{ C. parapsilosis $(n=3)$} \\
\hline $\mathrm{s}$ & 100 & 100 & 100 & 100 & 100 & 100 & 100 & 66.7 & 100 & 100 & 100 & 100 & 100 \\
\hline 1 & 0 & 0 & 0 & 0 & 0 & 0 & 0 & 33.3 & 0 & 0 & 0 & 0 & 0 \\
\hline $\mathrm{R}$ & 0 & 0 & 0 & 0 & 0 & 0 & 0 & 0 & 0 & 0 & 0 & 0 & 0 \\
\hline \multicolumn{14}{|l|}{ C. krusei $(n=2)$} \\
\hline $\mathrm{s}$ & 100 & 50 & 50 & 50 & 100 & 100 & 100 & 50 & 100 & 50 & 100 & 50 & 50 \\
\hline 1 & 0 & 0 & 0 & 50 & 0 & 0 & 0 & 0 & 0 & 50 & 0 & 0 & 0 \\
\hline $\mathrm{R}$ & 0 & 50 & 50 & 0 & 0 & 0 & 0 & 50 & 0 & 0 & 0 & 50 & 50 \\
\hline \multicolumn{14}{|l|}{ C. catenulata $(n=1)$} \\
\hline s & 100 & 100 & 100 & 100 & 100 & 100 & 100 & 100 & 100 & 100 & 100 & 100 & 100 \\
\hline 1 & 0 & 0 & 0 & 0 & 0 & 0 & 0 & 0 & 0 & 0 & 0 & 0 & 0 \\
\hline $\mathrm{R}$ & 0 & 0 & 0 & 0 & 0 & 0 & 0 & 0 & 0 & 0 & 0 & 0 & 0 \\
\hline
\end{tabular}

S: Susceptible, I: Intermediate, R: Resistant.

\begin{tabular}{|c|c|c|c|c|c|c|c|}
\hline \multicolumn{2}{|c|}{ Table 3: Antifungal resistant pattern of Candida albicans in-patient taking ART/ not with reference to their CD4 ${ }^{+}$count. } \\
\hline ART & ND4+ & Number of patients & FLU25 & FLU100 & ITC50 & POS5 & V01 \\
\hline & CD4+ & 21 & 52.4 & 52.4 & 47.6 & 47.6 & 52.4 \\
\hline NART & $<200$ & 13 & 76.9 & 76.9 & 42.9 & 61.5 & 69.2 \\
\hline & $200-500$ & 4 & 50 & 25 & 50 & 25 & 50 \\
\hline & $>500$ & 0 & & & & & \\
\hline OART & $<200$ & 12 & 58.3 & 58.3 & 50 & 41.7 & 66.7 \\
\hline & $200-500$ & 24 & 50 & 45.8 & 29.2 & 37.5 & 45.8 \\
\hline
\end{tabular}

\section{Discussion}

Little knew about the etiological importance of the species spectrum of different yeast species in the oral colonization and infection among Libyan HIV/AIDS patients. In this respect, it is the first Libyan study addressing Candida species and their antifungal susceptibility profiles to a wider spectrum of antifungal agents.in AIDS patients. Various studies have shown the isolation rate of Candida ranging from 61100 per cent in HIV infected individuals with oral candidiasis [16-19], while a wide range of oral colonization rates have been reported for asymptomatic HIV infected (11.0-96.0\%) as well for HIV uninfected (10.0-68.0\%) subjects [20]. This variability in colonization rates reported might caused by geographic differences, time of sampling or the different methods used for yeast recovery and quantitation [10]. We found a high prevalence of $C$. albicans recovered from HIV-infected and Aids patients with 74(74\%). In fact, this is by far the most prevalent commensal and pathogenic of the Candida 
species isolated in our early studies $[21,22]$. However, increasing rates of colonization and even infections with other species of Candida in HIV-infected individuals observed elsewhere [23]. Non-albicans species were isolated from 26 (26\%) patients. C. dubliniensis and C. glabrata were the most frequent non-albicans species recovered in this study. Of particular interest are the isolation and identification of $C$. dubliniensis as this is up to our knowledge the first formal report of the prevalence of $C$. dubliniensis in the Libyan population especially in HIV-infected individuals. Our results suggested that C. dubliniensis can be found quite frequently even in the healthy population. It found in our previous study to be the third most common species isolated in dental caries [22] and the second in HIV-infected patients in this study. Therefore, our data indicated that $C$. dubliniensis should not consider prevalent only in HIV-infected patients but also healthy individuals.

Our findings suggested that both antiretroviral therapy and CD4 T-lymphocyte count are important factors associated with low density of oral Candida colonization even in this group of relatively healthy HIV-infected patients. This is in concordance with other earlier studies that observed a higher rate of Candida colonization in patients with low CD4 counts [24]. The advent of ART has allowed for the suppression of viral replication to very low levels and a partial recovery of CD4+cells in patients with HIV, which has consequently reduced opportunistic infections as observed in our studies and others [25]. The low density of yeast oral colonization and candida infection even in patient harbor high oral yeast density found in our study may reflect the fact that the Libyan government currently guarantees free and universal access to HAART for all patients with HIV. Factors that could influence the oral colonization of Candida in patients with HIV and low count of CD4+cells as an important contributor to increase the probability of oral Candida colonization. A CD4+cell count less than 200 cells/ml considered a predisposing factor for oral candidiasis [6,7]. However, our results indicate that only a low CD4+cell count in patients not on HAART may be associated to progressing for high risk of oral Candida infection as it is indicated by high density oral colonization among most patient not on ART 32 out of $48(66.7 \%)$.

Several studies have shown a high prevalence of oral Candida colonization in HIVinfected individuals $[5,10,15]$. This different finding can vary according to sampling method and geographical location $[6,7,10]$. Using oral rinse for yeast isolation by other studies have shown to detected oropharyngeal Candida colonization in approximately $60 \%$ to $70 \%$ of HIV-infected individuals [10]. In our study, although $100 \%$ of HIVinfected individuals' were colonized by Candida species, the therapeutic combination of ART with exerted a protective effect were found to decrease yeast oral density in this population compared to patient not on ART as well as oropharyngeal Candida infections. The effect of highly active ART on the risk of Candida colonization in HIVinfected described elsewhere with a slight decrease [25]. Other authors also observed protective effect of ART treatment regimens [25-28]. On the other hand, higher oropharyngeal Candida colonization detected in ART-treated HIV infected individuals [29], have reported Oropharyngeal yeast colonization in patients receiving ART. Nevertheless, the data on the prevalence of Candida species in the oral cavity could be useful for initial treatment choice.

The important finding of the study was the high proportion of fluconazole, itraconazole, posoconazole and voriconazole-resistant among C. albicans. similar finding were observed by others [30,31], the previous use of azole agents was strongly associated with higher high fluconazole resistant against yeast isolates, making it more difficult to select an empiric therapy during the development of candidiasis in these patients as it is observed in our study. C. albicans in our study show high and cross resistant to fluconazole, itraconazole, posoconazole and voriconazole. This may attributed to both the high prevalence of the species as well as early use of other early azoles and fluconazole in Libyan hospital and community setting to treat other candida 
infections. Persistent asymptomatic colonization of Candida species is a possible risk factor for subsequent oral infection and a substantial set of epidemiological data should exist regarding the prevalence of Candida species in HIV positive individuals and its increase with disease progression are important tool to monitor and prevent oral pharyngeal candidiasis [31-33]. Few studies have performed about asymptomatic oral yeast colonization and its relationship to ART and CD4+count. In the present study, we did find a significant correlation between density of oral colonization and CD4+cell count or ART. These finding may be due to treatment guideline using efavirenz that inhibit the polymerization by altering the position of critical amino acids within the catalytic site and Combivir as a competitively inhibit and reduce the activity of reverse transcriptase [34]. This both drugs in turn causing HIV infected cells to decrease the number of viruses in the body and a partial recovery of CD4+cells in patients with HIV, which has consequently reduced opportunistic infections [28].

These results were similar to the studies by other study that reported a significant relationship between colonization rate and CD4+cell counts less than 200 cells $/ \mathrm{mm} 3$ [7]. Despite the fact that $27 \%(27 / 100)$ of the oral colonization had their CD4+cell count lower than 200 cells $/ \mathrm{mm} 3$ only one patient was on ART had lower CD4+count and harbor less than $100 \mathrm{CFU} / \mathrm{ml}$ candida among 27 patients. In contrast, among 26 patients not on ART 19 of them have more than $10^{5} \mathrm{CFU} / \mathrm{ml}$. These finding may support the important role of both ART and CD or either one of them in lowing density oral candida colonization and protection against oral candida infection in HIV patients, even in patients with more than $10^{5} \mathrm{CFU} / \mathrm{ml}$. Since only two patients in our study were with clinical oral thrush and dysphagia and the reaming patient have no clinical manifestation found. This may be due the fact there is no clear cut for oral density differentiate colonization and infection in our study as no clinical signs of oral candida infection among both patient with low or high oral density colonization.

\section{Conclusion}

Oral colonization of Candida spp. especially $C$. albicans was been detected with high frequency in HIV-infected patients. Early detection of oral colonization of Candida spp seem to be important for identification of patients with the propensity for rapid progression of HIV infection since oral colonization may influence the development of clinically significant candidiasis in these immunocompromised patients. We have also shown that the status of oral Candida carrier density is associated with both the number of CD4 cells and the ART.

\section{References}

1. William JD, Timothy BG. Andrews' diseases of the skin: Clinical dermatology. Saunders Elsevier. 2006; 45.

2. Crispian S. Oral and maxillofacial medicine: The basis of diagnosis and treatment ( $2^{\text {nd }}$ ed). Edinburgh: Churchill Livingstone. 2008; 191-199.

3. Fidel PL. Candida-host interactions in HIV disease: Relationships in oropharyngeal candidiasis. Adv Dent Res. 2006; 19: 80-84. Ref.: https://tinyurl.com/ybo4n8mn

4. Mousavi SAA, Salari S, Rezaie S, Nejad NS, Hadizadeh S, et al. Identification of Candida species isolated from oral colonization in Iranian HIV-positive patients, by PCR-RFLP method. Jundishapur $\mathrm{J}$ Microbiol. 2012; 5: 336-340. Ref.: https://tinyurl.com/ya6sa46j

5. Wabe NT, Hussein J, Suleman S, Abdella K. In vitro antifungal susceptibility of Candida albicans isolates from oral cavities of patients infected with human immunodeficiency virus in Ethiopia. $J$ Exper and Integra Med. 2011; 1: 265-271. Ref.: https://tinyurl.com/yak5a2w4

6. Khan AP, Malik A, Khan SH. Profile of candidiasis in HIV infected patients. Ira J of Microiol. 2012; 4 : 204-209. Ref.: https://tinyurl.com/ycpztjby

7. Vajpayee M, Kanswal S, Seth $P$, Wig N. Spectrum of Opportunistic Infections and Profile of CD4+Counts among AIDS Patients in North India. Infect. 2003; 31: 336-340. Ref.: https://tinyurl.com/ydxly745 
8. Martins MD, Lozano-Chiu M, Rex JH. Point prevalence of oropharyngeal colonization of fluconazoleresistant Candida in human immunodeficiency virus-infected patients. Clin Infect Dis. 1997; 25: 843846. Ref.: https://tinyurl.com/y7omeuku

9. Michelet C, Arvieux C, François C, Besnier JM, Rogez JP, et al. Opportunistic infections occurring during highly active antiretroviral treatment. AIDS. 1998; 12: 1815-1822. Ref.: https://tinyurl.com/ybw86yxp

10. Tooyama H, Matsumoto T, Hayashi K, Kurashina K, Kurita H, et al. Candida concentrations determined following concentrated oral rinse culture reflect clinical oral signs. BMC Oral Health. 2015; 15: 150. Ref.: https://tinyurl.com/ybdpow64

11. Jan A, Bashir G, Fomda BA, Khangsar DA, Manzoor M, et al. Hypertonic xylose agar medium: A novel medium for differentiation of Candida dubliniensis from Candida albicans. Indian J Med Microbiol. 2018; 35: 518-521. Ref.: https://tinyurl.com/y79ajhrq

12. Khan ZU, Ahmad S, Mokaddas E, Chandy R. Simplified sunflower (Helianthus annuus) seed agar for differentiation of Candida dubliniensis from Candida albicans. Clin Microbiol Infect. 2004; 10: 590592. Ref.: https://tinyurl.com/y75v62ww

13. Clinical and Laboratory Standards Institute (CLSI). Method for antifungal disk diffusion susceptibility testing of yeasts; approved guideline-2 ${ }^{\text {nd }}$ ed. CLSI document M44-A2. Wayne. PA: CLSI. 2009.

14. Clinical and Laboratory Standards Institute (CLSI). Zone diameter interpretive standards, corresponding minimal inhibitory concentration (MIC) interpretive breakpoints, and quality control limits for antifungal disk diffusion susceptibility testing of yeasts, Third International Supplement CLSI document-M44-S3. Wayne. PA: CLSI. 2009.

15. De Resende MA, de Sousa LV, de Oliveira RC, Koga-Ito CY, Lyon JP. Prevalence and Antifungal Susceptibility of Yeasts Obtained from the Oral Cavity of Elderly Individuals. Mycopathologia. 2006; 162: 39-44. Ref.: https://tinyurl.com/y9exh4v8

16. Wabale V, Kagal A, Bharadwaj R. Characterization of Candida spp. from oral thrush in human immunodeficiency virus (HIV) seropositive and seronegative patients. Bombay Hospital J. 2008; 50 212-217. Ref.: https://tinyurl.com/ya9j3esx

17. Baradkar VP, Kumar S. Species identification of Candida isolates obtained from oral lesions of HIV infected patients. Indian J Dermatol. 2009; 54: 385-386. Ref.: https://tinyurl.com/y9vrzx2s

18. Lattif AA, Uma B, Rajendra P, Biswas A, Wig N, et al. Susceptibility pattern and molecular type of speciesspecific Candida in oropharyngeal lesions of Indian human immunodeficiency virus-positive patients. J Clin Microbiol. 2004; 42: 1260-1262. Ref.: https://tinyurl.com/ycx525bg

19. Chunchanur SK, Nadgir SD, Halesh LH, Patil BS, Kausar Y, et al. Detection and antifungal susceptibility testing of oral Candida dubliniensis from human immunodeficiency virus-infected patients. Indian $\mathrm{J}$ Pathol Microbiol. 2009; 52: 501-504. Ref.: https://tinyurl.com/y9vco8s3

20. Campisi G, Pizzo G, Milici M, Mancuso S, Margiotta V. Candidal colonization in the oral cavity of human immunodeficiency virus-infected subjects. Oral Surg Oral Med Oral Path Oral Radiol Oral Endod. 2002; 93: 281-286. Ref.: https://tinyurl.com/y8ntoyka

21. Ellabib MS, ElJariny IA. In vitro activity of 6 antifungal agents on candida species isolated as causative agents from vaginal and other clinical specimens. Saudi Med J. 2001; 22: 860-863. Ref.: https://tinyurl.com/y9e51914

22. Krema ZA, Trfas EEM, Ellabib MS, Cogliati M. Oral colonization of candidiasis in patients with ora dental diseases: predisposing factors, species and their antifungal susceptibility patterns. Journal of Bacteriology \& Mycology: Open Access. 2018; 6: 223-227.

23. Arora U, Jagdev M, Jindal N. Immunosuppression level in HIV-positive patients with oropharyngeal Candidiasis. Indian J Med Microbiol. 2009; 27: 174-175. Ref.: https://tinyurl.com/yaxz8yom

24. Costa CR, de Lemos JA, Passos XS, de Araú jo CR, Cohen AJ, et al. Species distribution and antifungal susceptibility profile of oral Candida isolates from HIV-infected patients in the antiretroviral therapy era. Mycopathologia. 2006; 162: 45-50. Ref.: https://tinyurl.com/y76wryqg

25. EYESON JD, TENANT-FLOWERS M, COOPER DJ, NW Johnson, KAAS Warnakulasuriya. Oral manifestations of an HIV positive cohort in the era of highly active anti-retroviral therapy (HAART) in South London. J. oral Path. Med. 2002; 31: 169-174. Ref.: https://tinyurl.com/y855q52x

26. Staszewski S1, Loveday C, Picazo JJ, Dellarnonica P, Skinhøj P, et al. Safety and efficacy of lamivudine-zidovudine combination therapy in zidovudine-experienced patients. A randomized controlled comparison with zidovudine monotherapy. Lamivudine European HIV Working Group. JAMA. 1996; 276: 111-117. Ref.: https://tinyurl.com/ycnf6haw 
27. Nicolatou-galitis O, Velegraki A, Paikos S, Economopoulou P, Stefaniotis T, et al. Effect of pi-haart on the prevalence of oral lesions in HIV-I infected patients. A Greek study. Oral Dis. 2004; 10: 145-150. Ref.: https://tinyurl.com/y8mlnu29

28. Gugnani HC, Becker K, Fegeler W, Basu S, Chattopadhya D, et al. Oropharyngeal colonization of Candida species in HIV-infected patients in India. Mycoses. 2003; 46: 299-306. Ref.: https://tinyurl.com/y745fuqs

29. Yang YL, Lo HJ, Hung CC, Li Y. Effect of prolonged HAART on oral colonization with Candida and candidiasis. BMC Infect Dis. 2006; 6: 8. Ref.: https://tinyurl.com/yc6622f8

30. Sanchez-Vargas LO, Ortiz-Lopez NG, Villar M, Moragues MD, Aguirre JM, et al. Point prevalence, microbiology and antifungal susceptibility patterns of oral Candida isolates colonizing or infecting Mexican HIV/AIDS patients and healthy persons. Rev Iberoam Micol. 2005; 22: 83-92. Ref.: https://tinyurl.com/y92stslu

31. Vijeta Maurya, Ashutosh Srivastava, Jyoti Mishra, Rajni Gaind, Rungmei SK Marak, et al. Oropharyngeal candidiasis and Candida colonization in HIV positive patients in northern India. $\mathrm{J}$ Infect Dev Ctries. 2013; 7: 608-613. Ref.: https://tinyurl.com/ycy4nunm

32. Sangeorzan JA, Bradley SF, He X, Lidija Zarins T, George Ridenour L, et al. Epidemiology of oral candidiasis in HIV-infected patients: colonization, infection, treatment, and emergence of fluconazole resistance. Am J Med. 1994; 97: 339-346. Ref.: https://tinyurl.com/y8f3vqt3

33. Sánchez-Vargas LO, Ortiz-López NG, Villar M, María Dolores Moragues, José Manuel Aguirre, et al Oral Candida isolates colonizing or infecting human immunodeficiency virus-infected and healthy persons in Mexico. J Clin Microbiol. 2005; 43: 4159-4162. Ref.: https://tinyurl.com/yajt6aw9

34. Thomas Campbell B, Laura Smeaton M, Kumarasamy N, Timothy Flanigan, Karin Klingman L, et al. Efficacy and Safety of Three Antiretroviral Regimens for Initial Treatment of HIV-1: A Randomized Clinical Trial in Diverse Multinational Settings. PLOS Med. 2012; 9: e1001290. Ref.: https://tinyurl.com/ycty3qrh 